\title{
Diet and the metabolic syndrome: a cross-sectional study of 301 men from Stockholm County, Sweden
}

\section{Magdalena Rosell}

\section{Introduction}

The metabolic syndrome is a common condition that increases the risk of cardiovascular disease and type II diabetes. It is characterized by a clustering of disturbances such as insulin resistance, raised levels of plasma glucose, dyslipoproteinaemia, hypertension and a prothrombotic state. Obesity is an important contributor to the metabolic syndrome and particularly so when the body fat is accumulated in the abdominal region. The increasing prevalence of overweight and type II diabetes indicates that the metabolic syndrome will be a growing health problem in the future.

Although the pathogenesis of the metabolic syndrome is complex, from the point of view of prevention, the target risk factors are those associated with lifestyle in which dietary habits play an important role. However, nutritional research is complicated by the difficulties that arise when people's dietary habits are to be measured. Although underreporting of energy intake is common in dietary surveys, few studies take this into consideration when dietary intake is related to disease variables. The objective of the thesis was to study the role of diet in the metabolic syndrome with special reference to methodological issues related to the assessment of dietary intake.

\section{Methods}

The thesis was based on a cross-sectional study of 301 healthy men aged 63 years, conducted in 20002001 (1). The participants were recruited from a cohort of men who had attended a baseline investigation in 1998-1999 in Stockholm. They were recruited to be free of disease but to have a wide range of fasting insulin. The men underwent a medical examination including measurements of body weight, height, waist and sagittal abdominal diameter (SAD). Blood pressures were measured, fasting blood samples were drawn and a needle biopsy of adipose tissue was taken from the upper buttock. The participants' food intake was assessed using a 7 day precoded, optically readable food record. The booklet also contained space where the participants could record their intake in free text (the manually coded part). The reported intake of protein, sodium and potassium was validated using $24 \mathrm{~h}$ urine collections. Physical activity during the latest year was recorded in an interview and the physical activity level (PAL) was systematically estimated for each individual (1). According to the Goldberg equation, individual cut-offs were calculated for when the reported energy intake was too low to be due to chance. The men were divided by this means into underreporters (URs) and nonunderreporters (non-URs).

\section{Results and discussion}

In the present study, $29 \%$ of the participants were classified as URs. The prevalence of the metabolic syndrome was twice as high in the URs (18\%) compared with the non-URs (9\%) (1). The URs had a higher nutrient and food densities in their reported diet than did the non-URs, indicating that underreporting concerns specific types of foods. At a group level, the energy intake was underreported in both groups. However, there was a marked difference in the degree of underreporting; the ratio of energy intake:basal metabolic rate was 1.05 in the URs and 1.47 in the non-URs $(p<$ 0.001). The biological markers of protein, sodium and potassium confirmed a lower validity of the dietary data in the URs (1).

The importance of the manually coded part of the food record was also explored. On average, $22 \%$ of the reported energy intake came from this part (2). The manually coded part contributed predominantly food items typically eaten between meals, such as fruit, buns, sweets, snacks and desserts. The URs used the manually coded part to a lesser degree 
than the non-URs, which supports the hypothesis that food consumed between meals is more prone to be underreported than food consumed in main meals.

When the dietary intake was related to components in the metabolic syndrome, different results were seen in the URs and non-URs $(1,3)$. For example, in the URs, associations were seen between fasting insulin and the intake of polyunsaturated fat $(r=0.45, p<0.001)$ and also between the SAD and the intake of dairy fat $(r=-0.36, p=$ $0.001)$. These associations were not seen in the nonURs ( $r=0.07$ and $r=-0.04$, respectively). Regarding dairy fat, the study also explored the association between SAD and the relative content of the fatty acids 14:0, 15:0 and 17:0 in adipose tissue (AT) and serum phospholipids (PL) (these fatty acids are related to the intake of dairy fat), and inverse associations were seen. However, if there were a true association between dairy fat intake and $\mathrm{SAD}$, one would expect to detect this also among the non-URs. Although the URs on average had a higher SAD than the non-URs, this difference did not explain the different correlation in the URs and non-URs (3). The findings highlight the question of how to use and interpret dietary data in underreporters.

The study investigated whether the relative content of the fatty acids 14:0, 15:0 and 17:0 in AT and PL were also related to other factors, independently of dairy fat intake. The fatty acids, except for 14:0 in PL, were inversely associated with the intake of alcohol ( $r$ ranging between -0.21 and -0.52$)$ (4). They were also positively related with physical activity, although statistically significant trends were only seen for 14:0 in PL and 17:0 in AT. The findings indicate that the fatty acids should be interpreted with caution as markers for dairy fat intake.

In conclusion, this thesis demonstrates that a critical evaluation of what dietary data actually stand for must precede the drawing of any conclu- sions regarding possible relationships between diet and disease. It highlights an important issue that is of general concern for all those involved in nutritional research.

\section{Acknowledgements}

This study was supported by grants from the Swedish Council for Working Life and Social Research, the Swedish Heart and Lung Foundation, the Swedish Society for Medicine, the Swedish Medical Research Council, The Swedish Nutrition Foundation and Unilever Bestfoods.

\section{Articles in the thesis}

1. Rosell MS, Hellenius M-LB, de Faire U, Johansson GK. The associations between diet and the metabolic syndrome vary with the validity of the dietary data. Am J Clin Nutr 2003; 78: 84-90.

2. Rosell M, Hellénius M-L, de Faire U, Berglund L, Gustafsson I-B, Johansson G. The contribution of a manually coded part in a optically readable, precoded 7day food record to the intakes of energy, nutrients and foods. Scand J Nutr 2003; 47: 123-31.

3. Rosell M, Johansson G, Vessby B, Berglund L, de Faire $\mathrm{U}$, Hellenius M-L. Association between the intake of dairy fat and calcium and abdominal obesity. 2003. Manuscript.

4. Rosell M, Johansson G, Berglund L, Vessby B, de Faire $\mathrm{U}$, Hellenius M-L. The effect of alcohol and physical activity on the fatty acids 14:0, 15:0, and 17:0 in serum phospholipids and adipose tissue used as markers for dairy fat intake. 2003. Manuscript.

The article is based on the author's thesis, which was conducted at the Institute of Environmental Medicine, Karolinska Institutet, Stockholm, Sweden. Defended on Dec. 16, 2003. ISBN: 91-7349-703-7.

\footnotetext{
Magdalena Rosell

Institute of Environmental Medicine

Karolinska Institutet

Box 210

SE-I7I 77 Stockholm

Sweden

Tel: + 46852487498

E-mail: magdalena.rosell@imm.ki.se
} 